\title{
Navigating uncertainty
}

\author{
Helen Macdonald head of education
}

The BMJ

Thought leaders, creative types, and scientists may embrace uncertainty as a route to creativity, discovery, and fulfilment. But uncertainty is often ignored, feared, and hushed up in clinical medicine. Our editorialist Steven Hatch invites clinicians to stop trafficking in certitude, with diagnoses and research results that sound triumphantly final (doi:10.1136/bmj.j2180). If we sound certain we participate in self delusion and encourage it among our patients. If uncertainty is embraced, its admission "forms the starting point for a more open conversation between patient and clinician," he writes.

But uncertainty may not feel creative and liberating in day to day practice, and it can be a driver of excess and harm such as antibiotic prescribing for minor or viral infections. A quest to reduce uncertainty and improve outcomes for patients has a place at the heart of clinical research. In an editorial Anna Mae Scott and Chris Del Mar explain how doctors' uncertainty about acute lower respiratory tract infection can lead them to overprescribe antibiotics for fear of serious infection (doi:10. $1136 / \mathrm{bmj} . j 2398$ ). The authors of the linked research paper argue that trials of different antibiotic prescribing

strategies-immediate, delayed, or none-for acute infection are inadequately powered to supply data on the harms (doi:10. 1136/bmj.j2148). Their prospective cohort study of more than 28000 people aged over 16 presenting to primary care with acute lower respiratory tract infection finds that harms were uncommon among those who opted to avoid antibiotics. But irrespective of treatment strategy, around a fifth of patients consulted their doctor again about the illness.

The philosopher Bertrand Russell said that "when one admits that nothing is certain one must, I think, also add that some things are more nearly certain than others." Doctors need to be able to discuss degrees of certainty, including numbers, with their patients. Gerd Gigerenzer and Kai Kolpatzik report on the success of a German health insurer's use of a fact box developed by the US clinician researchers Lisa Schwartz and Steve Woloshin to present benefits and harms of drugs to patients (doi:10.1136/bmj.j2460). Information that is easy to read, visualise, and share may also benefit doctors seeking to understand the mass of numbers produced by research. At The $B M J$ we are working on a project with Schwartz and Woloshin to adapt fact boxes to appear in our education articles. With a similar aim, our Rapid Recommendations project (www.bmj. com/rapid-recommendations) aims to visualise absolute numbers that support clinical practice recommendations, based on systematic reviews and meta-analyses.

Finally, from facts to rhetoric. Those who are uncertain how to vote in the UK general election next month could read the feature by journalist Matthew Limb (doi:10.1136/bmj.j2467). He outlines how much funding the Conservatives, Labour, and the Liberal Democrats have pledged to health and social care. How certain these numbers are remains to be discovered. 